\title{
Using the Spanish national identity card in social networks
}

\author{
V. GAYOSO MARTÍNEZ*, Institute of Physical and Information Technologies \\ (ITEFI), Spanish National Research Council (CSIC), Madrid, Spain.
}

L. HERNÁNDEZ ENCINAS**, Institute of Physical and Information Technologies (ITEFI), Spanish National Research Council (CSIC), Madrid, Spain.

\author{
A. MARTÍN MUÑOZ ${ }^{\dagger}$, Institute of Physical and Information Technologies \\ (ITEFI), Spanish National Research Council (CSIC), Madrid, Spain.
}

\author{
R. DURÁN DÍAZ ${ }^{\dagger \dagger}$, Department of Automatics, University of Alcalá, Madrid, \\ Spain.
}

\begin{abstract}
The distinctive security features of the Spanish electronic national identity card, known as Documento Nacional de Identidad electrónico, allow us to propose the usage of this cryptographic smart card in an authentication framework that can be used during the registration and login phases of internet services where the validation of the user's age and real identity are key elements, as it is the case for example of the so-called social networks. Using this mechanism with NFC-capable devices, the identity and age of the potential user can be determined, allowing or denying the access to the service based on that information.
\end{abstract}

Keywords: Authentication, DNIe, ROCA, smart cards, social networks

\section{Introduction}

The rapid development in our society of internet and other information technologies faces significant challenges, such as how to provide the proper mechanisms in order to guarantee the valid authentication of individuals and the privacy of the data transmitted. One way to perform the authentication of citizens is through the use of national identity smart cards, but only if they satisfy the proper physical and logical security levels.

The use of cryptographic protocols specifically designed for this type of smart card would allow users to authenticate themselves in a secure way. With this critical security layer, new services could be offered that would grant or deny the access to the requesting citizen based on his/her age or on the possibility of clearly identifying him/her.

Child protection is a paramount requirement nowadays, as the easy access to the new technologies makes kids a particularly vulnerable group. In this sense, news about child abuse through the

\footnotetext{
*E-mail: victor.gayoso@iec.csic.es

**E-mail: luis@iec.csic.es

†E-mail: agustin@iec.csic.es

${ }^{\dagger \dagger}$ E-mail: raul.duran@uah.es
}

Vol. 28, No. 4, (C) The Author(s) 2019. Published by Oxford University Press. All rights reserved. For permissions, please e-mail: journals.permission@oup.com. 
networks, especially the so-called social networks, are unfortunately very frequent in the developed countries. Some of the reasons for these abuses are their poor preparation for the potential risks they can encounter, the attempt to bypass certain limits, and the lack of attention of their parents or tutors.

Although some national and international initiatives have been announced in the past (e.g. [8] and [27]), in the interim it would be highly beneficial to develop new technologies, frameworks, protocols and applications that provide a reliable authentication and ensure the protection of children when using internet.

In this contribution, we present an authentication mechanism that employs the Spanish electronic identity card (known as DNIe, Documento Nacional de Identidad electrónico) as the core of a security framework that can be employed for authentication purposes in internet services where the user's age and real identity are primary factors for either signing up or allowing the login of individuals. In this scope, social networks represent a good example of that kind of service, as a minimum age is required before signing up in most social networks. The user experience of the proposed framework is improved when using the DNIe 3.0, which includes NFC (Near Field Communications) technology, removing the need to use a detached smart card reader.

This paper is organized as follows: Section 2 summarizes the basic knowledge about smart cards that is necessary for understanding some parts of this contribution. Sections 3 and 4 present the main features of the DNIe 2.0 and 3.0, respectively. Section 5 describes the certification process of the DNIe and the most important attack against the security of that card known so far. Section 6 describes the proposed authentication scheme that takes advantage of the distinctive features of the DNIe. Finally, in Section 7 we provide the conclusions of this work.

\section{Smart card concepts}

ISO/IEC 7816, managed jointly by ISO (International Organization for Standardization) and IEC (International Electrotechnical Commission) [15], is the most important set of smart card standards. The basic characteristics of smart cards are specified in ISO/IEC 7816-1 (physical requirements, [10]), 7816-2 (dimensions and location of the contacts, [11]), 7816-3 (electrical interface and transmission protocols, [12]) and 7816-4 (organization, security and commands for information interchange, [13]). The remaining parts specify other non-basic and optional characteristics.

Following the ISO/IEC 7816 notation, the smart card file structure is represented by means of two types of elements: DF (Dedicated File) and EF (Elementary File). While DFs can be interpreted as directories or folders of a standard file system, EFs can be considered to be data files, belonging either to the operating system or to other smart card applications.

The most widely used smart card communication protocol is the byte oriented protocol $\mathrm{T}=0$, which uses APDU (Application Protocol Data Unit) elements. APDUs are data packets that are exchanged between an external application and the smart card. The card operating system is responsible for analysing any APDU received and routing it to the proper application. It is also responsible for collecting the application response and submitting it to the card reader (and thus to the application that communicates with the reader).

Once the smart card is powered on by the card reader, its first action consists in sending the ATR (Answer To Reset), a string of bytes with information about technical aspects of the communication (clock frequency, maximum current, etc.) and proprietary data from the card manufacturer. After the initial details of the communication have been agreed, the smart card reader can start sending APDU commands, to which the smart card will answer with the response APDUs. 

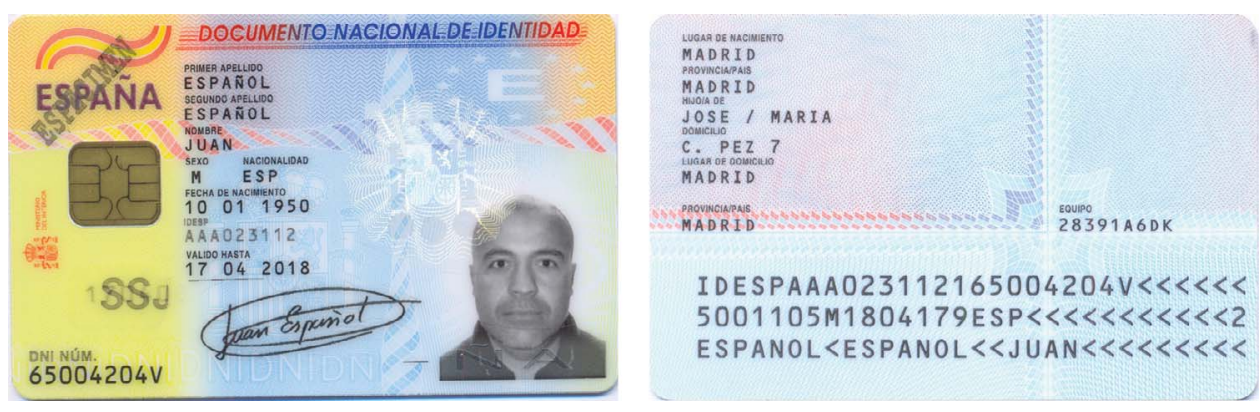

FIGURE 1. Front and back sides of a DNIe 2.0.

\section{DNIe 2.0}

The DNIe is a smart card that allows to certify the identity of the DNIe holder and to digitally sign documents using electronic signature protocols with the same legal validity than a handwritten signature [7]. The current Spanish legislation states that it is mandatory to obtain the DNIe for Spanish citizens over 14 years, as it is the preferred method to prove one's own identity in any act with the Public Administration. Regarding the terminology used in this work, the terms 'child' and 'minor' refer to citizens under 14 years, 'youngster' refers to citizens whose age goes from 14 to 18 years, and 'adults' refers to the rest of the population.

The DNIe started to be issued in 2006. Shortly after that, a version with a new operating system was launched using the name DNIe 2.0. More than 63 million of DNIe cards have been issued so far [18], so the installed base is certainly remarkable. Figure 1 shows the front and back sides of a DNIe 2.0 .

On top of the ISO/IEC 7816 file structure, all versions of the DNIe implement a subset of the PKCS \#15 (Public-Key Cryptography Standards) file scheme. PKCS \#15 is a standard that was initially released by Rivest, Shamir, \& Adleman (RSA) Laboratories [24] and, since January 2004, it is published as the ISO/IEC 7816-15 specification [14]. In brief, PKCS \#15 allows users of cryptographic devices to communicate with external applications regardless of the implemented API (Application Programming Interface), as the standard includes descriptions of the cryptographic objects stored in the device, as well as the ASN.1 (Abstract Syntax Notation One) syntax used to encode the content of such objects.

Figure 2 (left) shows the file tree of the DNIe 2.0, where the Master File (typically represented as the DF 3F00) is the root directory of the file system. Some of the most relevant EFs are listed below together with the requirements for accessing each file:

- EF 2F03: Information about the DNIe operating system (free access).

- EF 6004: Information about the certificates managed by the PKCS \#15 application (free access).

- EF 7001: Citizen's filiation data (name, surname, date of birth, etc.). This information is also printed on the front and back sides of the DNIe (biometric verification required).

- EF 7002: Digitized image of the citizen's photograph (biometric verification required).

- EF 7003: Digitized image of the citizen's handwritten signature (biometric verification required).

- EF 7004: X.509 user signing certificate with an RSA public key of 2048 bits (PIN code validation required). 

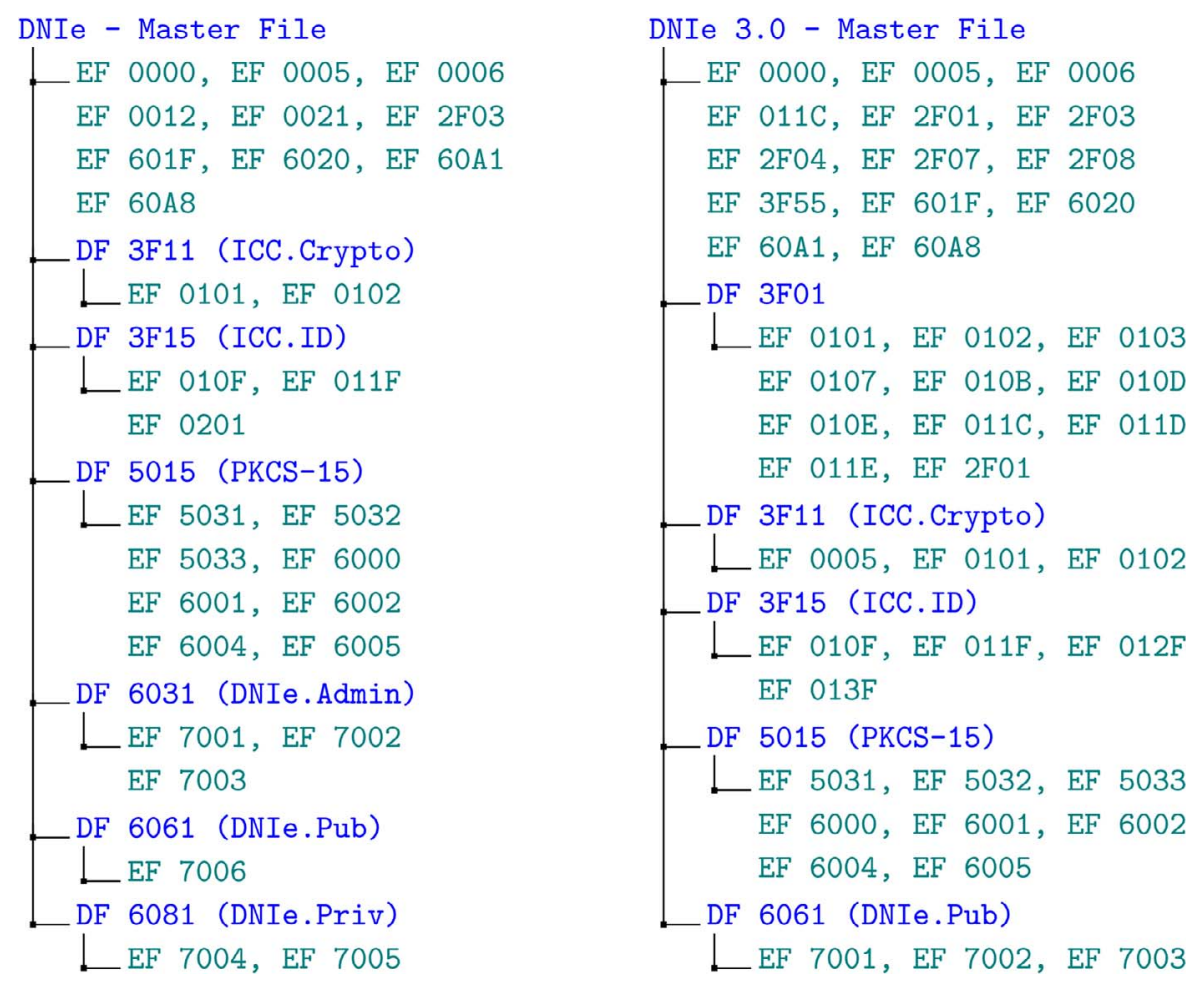

FIGURE 2. File structure of the DNIe 2.0 (left) and DNIe 3.0 (right).

- EF 7005: X.509 user authentication certificate with an RSA public key of 2048 bits (PIN code validation required).

In the scope of DNIe 2.0, although minors could legally request the DNIe, the card did not include any personal certificates. In comparison, the DNIe of youngsters could contain the authentication and signature certificates, but only with the authorization of their parents. Finally, all adults were issued both the signature and authentication certificates.

The DNIe is a SSCD (Secure Signature Creation Device) compliant with the European standard EN 14890-1 [3], which defines how to establish a communication between the SSCD and an external application. Because of that, the operating system of the DNIe 2.0 subordinates the sending of certain APDUs (like the Verify PIN command) to the establishment of a secure channel [17].

In order to establish the secure channel, it is necessary to exchange the public keys of the card and the external application that wants to communicate with the DNIe. After the certificates containing those keys are verified by both parties, they must perform a mutual authentication protocol, including a seed exchange for the derivation of the encryption and MAC (Message Authentication Code) session keys. Once the secure channel is established, any command must be protected before its transmission using the session keys. Readers interested in the specific details of the secure channel procedure are advised to read [2] and [9] in order to obtain more details. 

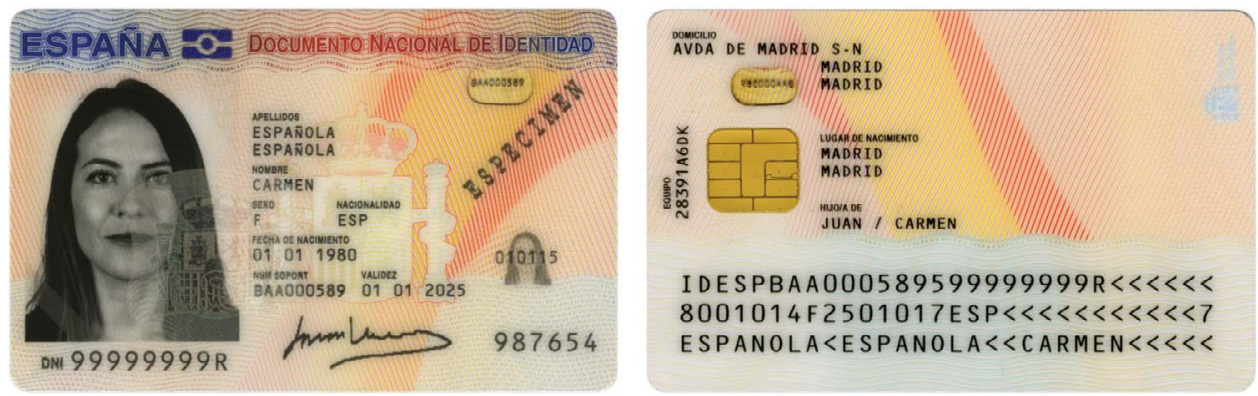

FIGURE 3. Front and back sides of a DNIe 3.0.

\section{DNIe 3.0}

In January 2015, a new version called DNIe 3.0 was presented (see Figure 3). The major novelty introduced in this version is the inclusion of an NFC chip with the goal to facilitate its usage with smartphones and tablets, avoiding the limitation of delivering smart card readers to potential users [19].

NFC is a set of protocols that enable two electronic devices to establish communications by bringing them to a close distance (typically between 1 and 10 centimetres). NFC devices can work in three modes [23]: card emulation, which enables NFC devices such as smartphones to act like smart cards, allowing users to perform transactions such as payment or ticketing; reader/writer, which enables NFC devices to act like smart card readers, retrieving the information stored on NFC tags embedded in labels or smart posters; and peer-to-peer, which enables two NFC devices to communicate with each other to exchange information. The DNIe 3.0 can communicate with NFC-capable devices using the card emulation mode.

Figure 2 (right) shows the file tree of the DNIe 3.0. Some of the most relevant EFs are the following ones, where all those files can be read without validating the personal identification number (PIN):

- EF 0102: Digitized image of the citizen's photograph.

- EF 0107: Digitised image of the citizen's handwritten signature.

- EF 010D: Citizen's filiation data (name, surname, date of birth, address, etc.).

- EF 2F03: Information about the DNIe operating system.

- EF 2F08: CAN (Card Access Number) needed for NFC pairing.

- EF 6004: Information about the certificates managed by the PKCS \#15 application.

- EF 7001: X.509 user authentication certificate with an RSA public key of 2048 bits.

- EF 7002: X.509 user signing certificate with an RSA public key of 2048 bits.

The DNIe 3.0 maintains the prohibition that does not allow to send the Verify APDU command in clear, but introduces as a novelty a second secure channel specifically for sending that command. Even though the procedure is the same in comparison with the secure channel used in the DNIe 2.0 (with the sole exception that the MAC values generated by the DNIe 3.0 are 8 bytes long while the MAC values generated by the DNIe 2.0 only had 4 bytes), some of the values used in the procedure have changed. Those values are the public key, private key and certificate of the terminal (i.e. the software application that intends to communicate with the DNIe), and the internal references used to manage those elements. 
As it has been previously mentioned, another change introduced by the DNIe 3.0 is the lack of PIN validation when accessing certain files that store personal information, such as the user's certificates, the filiation data or the digitized images associated to the citizen and his/her signature. Even though in order to read all those files it is necessary to establish the secure channel, as it is not necessary to validate the user PIN for reading those files, any application accessing the DNIe can retrieve that information. From our point of view, that constitutes a threat to the user's privacy if a rogue application has access to the smart card.

\section{DNIe security}

Smart card security is often described by means of the Evaluation Assurance Level (EAL) obtained by the card following the completion of a Common Criteria security evaluation. EAL levels range from EAL1 to EAL7 [6]:

- EAL1: Functionally tested.

- EAL2: Structurally tested.

- EAL3: Methodically tested and checked.

- EAL4: Methodically designed, tested, and reviewed.

- EAL5: Semiformally designed and tested.

- EAL6: Semiformally verified, designed and tested.

- EAL7: Formally verified, designed and tested.

In some cases, the evaluation may be augmented to include assurance requirements beyond the minimum required for a particular EAL. Those cases are typically denoted with a plus sign (e.g. EAL4+).

The software of the DNIe 2.0 received the EAL4+ AVA_VAN.5 accreditation in November 2015 [25], whereas the software of the DNIe 3.0 was certified with the same accreditation level in February 2017 [26]. The term AVA_VAN refers to the vulnerability assesment level achieved by the device, which is graded in the following way [6]:

- AVA_VAN.1: Vulnerability survey (TOE is resistant to an attacker possessing basic attack potential, where TOE stands for Target of Evaluation).

- AVA_VAN.2: Vulnerability analysis (TOE is resistant to an attacker possessing basic attack potential)

- AVA_VAN.3: Focused vulnerability analysis (TOE is resistant to an attacker possessing enhanced-basic attack potential).

- AVA_VAN.4: Methodical vulnerability analysis (TOE is resistant to an attacker possessing moderate attack potential).

- AVA_VAN.5: Advanced methodical vulnerability analysis (TOE is resistant to an attacker possessing high attack potential).

It must be understood that a product that is EAL4+ AVA_VAN.5 might be practically more secure than a product that is 'simply' EAL5, since EAL5 only requires AVA_VAN.4. It must also be noted that, apart from the operating system, the chips used in smart cards also apply for their own security certification.

In spite of the reassurance provided by EAL certifications, sometimes design errors or faulty implementations go undetected and are present in security products already deployed in the field. That was the case of one of the chips used by the DNIe; more precisely, the weakness was located 
in the Infineon RSA library version 1.02.013 that was included in the SLE78CLFX1600P chip used by the DNIe.

In October 2017, researchers Matus Nemec, Marek Sys, Petr Svenda, Dusan Klinec and Vashek Matyas, from the Masaryk University, announced that they had discovered a vulnerability in the generation of RSA keys used by Infineon's software library. They gave the name ROCA (Return of Coppersmith's Attack) to their attack, which is feasible against the most commonly used key lengths, including 1024 and 2048 bits, and affects chips manufactured as early as 2012, according to the authors of the attack [5].

The algorithmic vulnerability is characterized by a specific structure of the generated RSA primes, which makes factorization of some key lengths possible in practice. The specific structure of the primes in question allows for a fast detection of vulnerable keys, and in fact the authors published the code of an application that was able to detect if a specific certificate was vulnerable in several programming languages [4]. The pseudocode included in Algorithm 1, which is based on the code developed by Matus Nemec et al. and posted on [4], shows how to check if the modulus of an RSA certificate is vulnerable or not, where the symbols $\%$ and $\&$ represent the modulo and the bitwise AND operations, respectively.

According to the authors' computations, the average cases for the factorization of 1024 and 2048bit keys were less than 2 Central Processing Unit (CPU)-months and 50 CPU-years, respectively, on a single core central processing unit (CPU). In case of using cloud computing services such as Amazon $\mathrm{C} 4$ [1], the estimated cost for retrieving the private key in less than a few days was less than 100 and 40,000 dollars for 1024 and 2048 key lengths, respectively.

On 8 November 2017, the Spanish Ministry of Interior announced that, due to the discovery of the vulnerability, all certificates issued for DNIes whose support number was greater than ASG160.000 were revoked [21]. This measure affected all DNIes issued since April 2015. Besides, as a temporary solution, new DNIes would be emitted with RSA keys of 1920 bits, as that bit length seemed not to be affected by the attack. After applying the necessary technical solutions, the notification of the possibility of renewing the certificates was made on 1 December 2017 [20]. Thus, it can be stated that, unless another security attack is made public in the future, the DNIe is a secure platform without active threats against its security.

\section{Proposed framework}

During our research, we have identified several distinctive features presented by both the DNIe 2.0 and 3.0 that can be used by security applications. As our proposed framework uses some of those features, it is necessary to describe them before presenting the framework itself:

- The ATR that the DNIe sends after being powered on is a string of bytes that includes the term 'DNIe'.

- A DNIe 2.0 is identified by the value $0 \times 01$ located at the 16 th byte of the ATR, while a DNIe 3.0 is identified by the value $0 \times 04$ stored at that location.

- Minors can be identified by analysing the content of the EF 6004. If that file does not exist or does not include a reference to the user's signature certificate, then the DNIe belongs to a minor. It is important to recall that minors with a DNIe 2.0 do not have certificates of any kind, while minors with a DNIe 3.0 only have authentication certificates. In both cases, they lack the signature certificate, and that feature can be used for identifying minors, though in the case of the DNIe 3.0 there is another method for accomplishing the same task (i.e. reading the content of the EF 010D). 


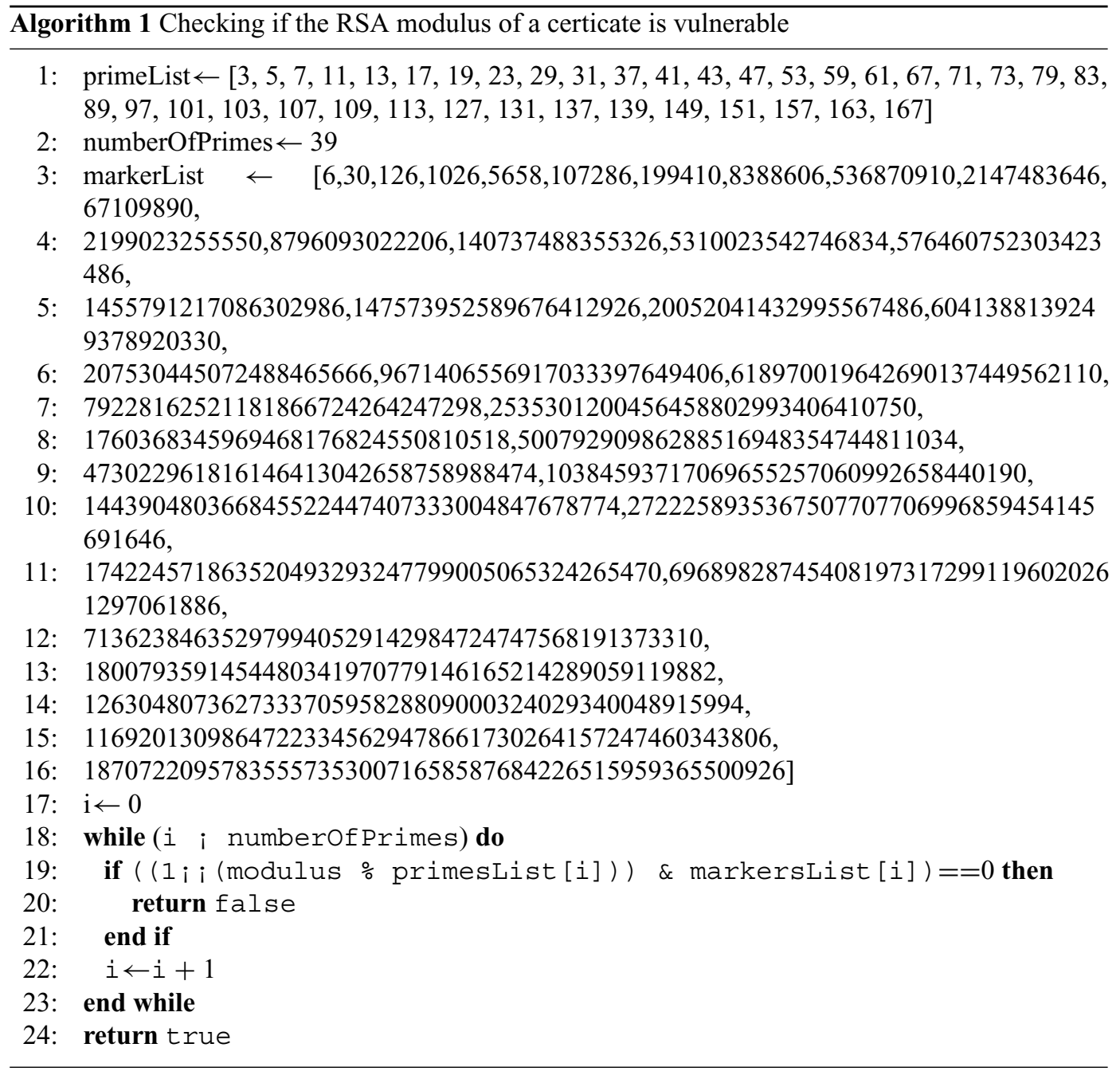

- The personal information that can be accessed in any DNIe (except in those belonging to minors possessing a DNIe 2.0) without performing the Personal Identification Number (PIN) verification is the following: country, name, and the sequence of 8 digits and a letter (which is computed by using a simple algorithm over the sequence of numbers [22]) associated to the individual. Those attributes correspond to elements included in the EF 6004 (and also in the user's signature and authentication certificates).

- The birth date of an adult with a DNIe 2.0 can be read from the user certificates only if they include the optional extension 2.5.29.9 after validating the PIN code [16]. In comparison, the birth date of any citizen having a DNIe 3.0 can be obtained without the user being requested to enter the PIN code. In both cases, it is necessary to establish the secure channel.

The proposed authentication framework that can be used for accessing internet services such as social networks is graphically depicted as a flow diagram in Figure 4. Once the DNIe is inserted 


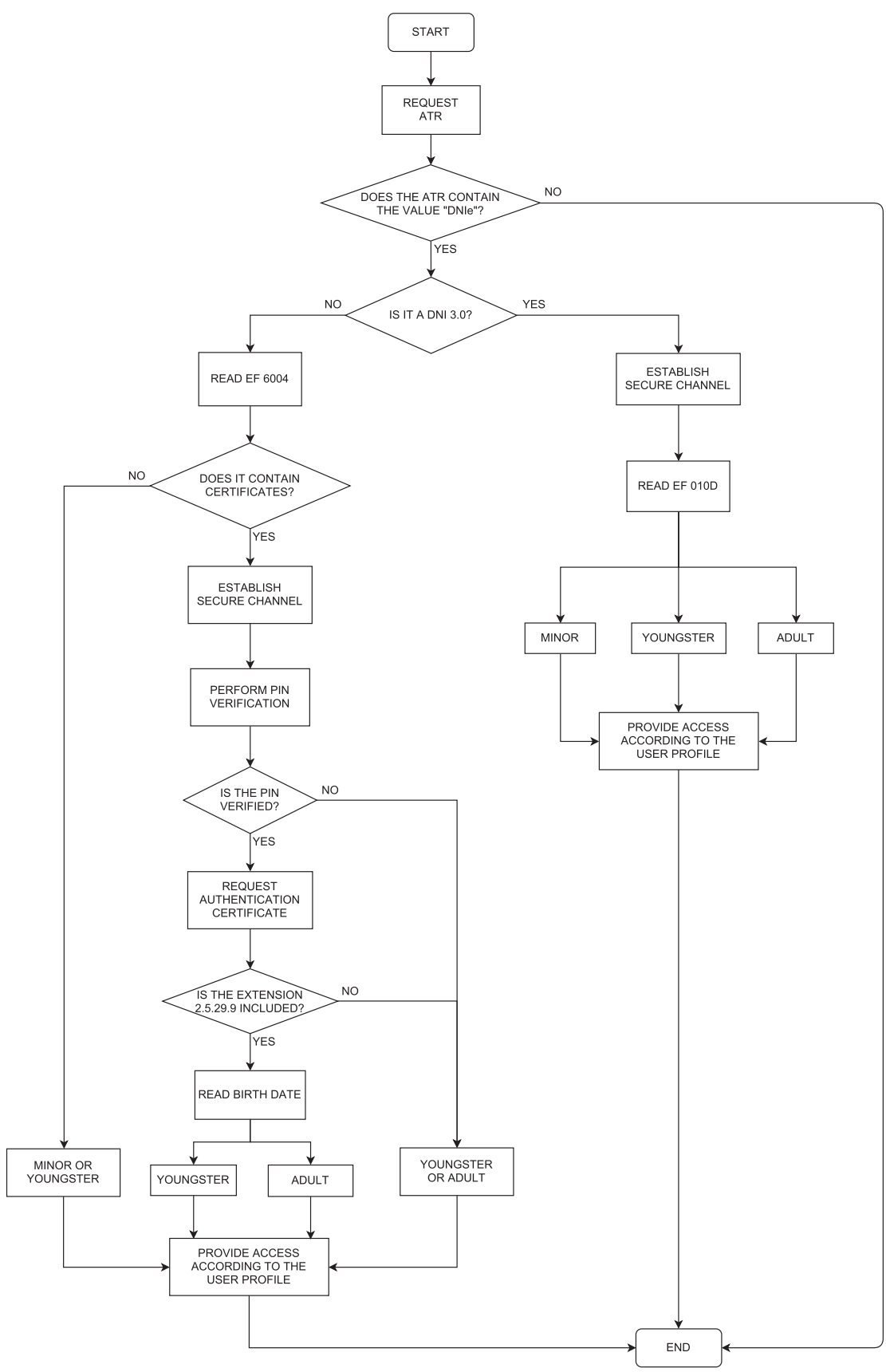

FIgURE 4. Proposed authentication framework. 
into a smart card reader or placed next to an NFC-capable device, the steps that must be completed during the operation of the protocol are the following ones:

1. The application initiates the process requesting the ATR of the smart card. This can be achieved by various means, e.g. using a method of the API that provides that information or programmatically resetting the DNIe.

2. The application checks if the ATR contains the hexadecimal string representing the term 'DNIe'. If that is not the case, it terminates the procedure showing an error message to the user.

3. If the smart card inserted in the reader is a DNIe, then the application identifies its type (either 2.0 or 3.0 ) by means of the 16 th byte of the ATR.

4. If the smart card inserted in the reader is a DNIe 2.0, then the application needs to read the EF 6004.

1. If the EF 6004 does not exist or it is empty (i.e. all its bytes have the value 0x00) then the DNIe belongs to a child or to a youngster without certificates. This fact can be used for denying access to users under the age of 18 .

2. On the other hand, if the EF 6004 is not empty, then the authentication protocol establishes a secure channel before trying to validate the user PIN. If the PIN is not validated (e.g. because the user cancels the validation process or the PIN code gets blocked), the application will only be able to determine if the user is either a minor or a youngster/adult.

3. If the PIN code is correctly validated, the application then reads the authentication certificate located at the EF 7005 and verifies if the extension with OID (Object Identifier) 2.5.29.9 is included in the certificate. If that is the case, it will be possible to determine the birth date of the user. However, if that extension is not included, then the application will only be able to determine if the user is either a minor or a youngster/adult.

5. If the smart card inserted in the reader is a DNIe 3.0, the protocol will read the file EF 010D in order to determine the birth date of the user, so it can provide him/her the proper service according to his/her age in all the cases.

Using this framework, applications can determine if a smart card is actually a DNIe and, depending on the type of DNIe and the user actions, they can discriminate if the DNIe belongs to a minor, a youngster or an adult, allowing access to the service or banning its usage.

This framework can be easily implemented in PC platforms, either as an independent Java application or as a service running in a web browser, and also as an Android application.

\section{Conclusions}

In this contribution, we have presented an authentication mechanism based on the usage of a national identity smart card. Even though the framework has been created with the Spanish DNIe in mind, the cryptographic similarities with the national identity smart cards from other countries (e.g. Italy, Finland, Estonia or Indonesia) would facilitate to extend the scheme to services from those countries.

Using the proposed framework and the DNIe 3.0, internet services requiring either the actual age or identity of users for signing up or during the login phase (as it is the case in many social networks) can determine if the accessing user is a child, a youngster or an adult. When using the older DNIe 2.0 , in some cases the protocol would only allow to determine if the citizen is not an adult (i.e. his/her age is below 18 years) or is not a child (i.e. his/her age is at least 14 years), depending on the actual DNIe used. With the capabilities provided by the proposed framework, internet services can decide 
either to accept the potential user or filter the content provided to him/her, protecting minors in this way from inappropriate content.

The rollout of the DNIe 3.0 allows to improve the user experience of authentication services, as it allows to use NFC-capable handsets instead of having to plug a smart card reader into a PC or handset. However, due to its design, this version of the DNIe permits applications to gain access to personal information, such as the citizen's digitized photograph and signature or its filiation data. We believe that those elements should be protected by the user PIN in order to prevent rogue applications from obtaining that information and using it without the knowledge of the citizen.

\section{Acknowledgements}

This research has been partially supported by Ministerio de Economía, Industria y Competitividad (MINECO), Agencia Estatal de Investigación (AEI) and Fondo Europeo de Desarrollo Regional (FEDER, UE) under project COPCIS, reference TIN2017-84844-C2-1-R, and by the European Union program ERASMUS+ under the project 2017-1-ESO1-KA203-038491 (Rules_Math).

\section{References}

[1] Amazon Web Services, Inc. C4 instances, featuring the highest compute performance on Amazon EC2, 2018. https://aws.amazon.com/es/about-aws/whats-new/2015/01/11/amazonec2-c4-instances-now-available/.

[2] D. Arroyo Guardeño, V. Gayoso Martínez, L. Hernández Encinas and A. Martín Muñoz. Using smart cards for authenticating in public services: a comparative study. In International Joint Conference: CISIS'15 and ICEUTE'15, Springer International Publishing Switzerland, pp. 437-446, 2015.

[3] CEN Application interface for smart cards used as secure signature creation devices. Part 1: basic services. European Committee for Standardization, 2009. UNE-EN 14890-1. http:// www.cen.eu/cen/Sectors/TechnicalCommitteesWorkshops/CENTechnicalCommittees/Pages/ Standards.aspx?param $=6205 \&$ title $=$ CEN

[4] Centre for Research on Cryptography and Security ROCA detection tool, 2017. https://crocs. fi.muni.cz/public/papers/rsa_ccs17.

[5] Centre for Research on Cryptography and Security ROCA: vulnerable RSA generation, 2017. https://github.com/crocs-muni/roca.

[6] Common Criteria Common criteria for information technology security evaluation. Part 3: security assurance components, 2017. https:/www.commoncriteriaportal.org/files/ccfiles/ CCPART3V3.1R5.pdf.

[7] J. Crespo Sánchez, J. Espinosa García, L. Hernández Encinas, H. Rifá Pous and M. Torres Hernández. Hacia una nueva identificación electrónica del ciudadano: el DNI-e. In Proceedings of IX Reunión Española de Criptología Y Seguridad de la Información (IX RECSI), UOC Press, pp. 660-673, 2006.

[8] European Commission European strategy for a better internet for children, 2012. http://eurlex.europa.eu/LexUriServ/LexUriServ.do?uri=CELEX:52012DC0196:EN:NOT.

[9] V. Gayoso Martínez, L. Hernández Encinas, A. Martín Muñoz, M. A. Álvarez Mariño and D. Arroyo Guardeño. A comparative study of three Spanish eGoverment smart cards. Logic Journal of the IGPL, 25, 2017.

[10] ISO/IEC Identification cards - Integrated circuit cards - Part 1: Cards with contacts — Physical 
characteristics. In International Organization for Standardization/International Electrotechnical Commission. ISO/IEC 7816-1, 2nd edn., ISO/IEC, 2011.

[11] ISO/IEC Identification cards-Integrated circuit cards-Part 2: Cards with contactsDimensions and location of the contacts. In International Organization for Standardization/International Electrotechnical Commission. ISO/IEC 7816-2, 2nd edn., ISO/IEC, 2007.

[12] ISO/IEC Identification cards-Integrated circuit cards-Part 3: Cards with contactsElectrical interface and transmission protocols. In International Organization for Standardization/International Electrotechnical Commission. ISO/IEC 7816-3, 3rd edn., ISO/IEC, 2006.

[13] ISO/IEC Identification cards - Integrated circuit cards - Part 4: Organization, security and commands for interchange. In International Organization for Standardization/International Electrotechnical Commission. ISO/IEC 7816-4, 3rd edn., ISO/IEC, 2013.

[14] ISO/IEC Identification cards - Integrated circuit cards-Part 15: Cryptographic information application. In International Organization for Standardization/International Electrotechnical Commission. ISO/IEC 7816-15, ISO/IEC, 2004.

[15] ISO/IEC Joint technical Comittee (JTC) 1/sub-comittee (SC) 17. In International Organization for Standardization/International Electrotechnical Commission, 2018. https://www.iso.org/ committee/45144.html.

[16] Ministerio del Interior de España Guía para desarrolladores con el DNIe electrónico, 2007. https://www.incibe.es/extfrontinteco/img/File/intecocert/dnie/pdf/guiades.pdf.

[17] Ministerio del Interior de España Documento Nacional de identidad electrónico: guía de referencia técnica, 2012. https://www.dnielectronico.es/PDFs/Guia_de_referencia_basica_v1_ 4.pdf.

[18] Ministerio del Interior de España Portal oficial sobre el DNI electrónico, 2013. http://www. dnielectronico.es/.

[19] Ministerio del Interior de España Guía de referencia del DNIe con NFC, 2015. http://www. dnielectronico.es/PDFs/Guia_de_Referencia_DNIe_con_NFC.pdf.

[20] Ministerio del Interior de España DNIe electrónico-Noticias, 2017. https://www. dnielectronico.es/PortalDNIe/.

[21] Ministerio del Interior de España Seguridad de los certificados electrónicos del DNIe, 2017. http://www.interior.gob.es/prensa/noticias/-/asset_publisher/GHU8Ap6ztgsg/content/id/ 7974097.

[22] Ministerio del Interior de España Cálculo del dígito de control del NIF/NIE, 2018. http://www. interior.gob.es/en/web/servicios-al-ciudadano/dni/calculo-del-digito-de-control-del-nif-nie.

[23] NFC Forum What is NFC, 2017. http://nfc-forum.org/what-is-nfc/.

[24] RSA Laboratories PKCS \#15: cryptographic token information syntax standard, 2000. ftp:// ftp.rsasecurity.com/pub/pkcs/pkcs-15/pkcs-15v1_1.pdf.

[25] F. Sanz Roldán. DNIe 2.0 certification report, 2015. https://www.dnielectronico.es/PDFs/ EAL4_AVA_VAN.5.pdf.

[26] F. Sanz Roldán. DNIe 3.0 certification report, 2017. http://www.boe.es/boe/dias/2017/02/02/ pdfs/BOE-A-2017-1096.pdf.

[27] The Wall Street Journal Facebook explores giving kids access, 2012. https://www.wsj.com/ articles/SB10001424052702303506404577444711741019238.

Received 4 July 2018 